\title{
OMEP Position Paper: Early Childhood Education and Care in the Time of COVID-19
}

\author{
OMEP Executive Committee, World Organisation for Early Childhood \\ Education ${ }^{1}$
}

Published online: 3 August 2020

(c) Springer Nature B.V. 2020

The International Journal of Early Childhood (IJEC) is published under the auspices of OMEP (World Organisation for Early Childhood Education). The aims of OMEP are to promote the rights of the child with a special emphasis on rights to education and care, worldwide. OMEP holds special consultative status with the UN and UNESCO.

In the difficult situations engendered by the Coronavirus Pandemic in 2020, OMEP has special concerns for children's well-being. The global crisis has tested the political, health, and economic systems of many countries. In response, it is important to seek solutions through solidarity to help to prevent the spread of the virus and reduce its negative impacts for children and their families.

The OMEP Executive Committee has formulated a position paper: Early Childhood Education and Care in the Time of COVID-19. This position paper is presented below in English, French, and Spanish which are the three official languages of OMEP.

\section{Early Childhood Education and Care in the Time of COVID-19}

The early years are of vital importance, and State parties need to take special measures to protect children and to promote good living conditions based on the best interests of the child, in accordance with the provisions of the Convention on the Rights of the Child (UNCRC). In this time dominated by the global pandemic, there are additional risks for young children. The pandemic is not only a virus, it is a huge human crisis and it is verified that children living in conditions of poverty, extreme poverty, and inequality have worsened their situation.

There are also indirect consequences that affect deeply children's lives and development, such as family deaths, fear, confinement, hunger, physical

OMEP Executive Committee, World Organisation for Early Childhood Education worldpresidency@worldomep.org

1 OMEP World Organisation for Early Childhood Education, Buenos Aires, Argentina 
estrangement, increased violence and abuse, lack of moments for play and movement, and excessive exposure to screens.

Although the contexts and everyday life for young children are quite different, OMEP wants to highlight certain crucial points in the best interests of all children and their well-being around the world.

State parties are guarantors of the fulfillment of the Rights of the Child and have the duty to implement the necessary policies to protect and support children and families, in collaboration with local authorities and organizations. Early Childhood Education and Care (ECEC) plays a fundamental role in this support.

- Young children are citizens and State parties must also consider their specific needs in the context of education, care, health, economy, and social support.

- Young children require the adoption of specific measures within the framework of the UNCRC. Each child has a right to express his or her views freely in all matters affecting him/her, and to have them considered. Their ideas, emotions, perspectives, interests, and needs must be considered within the framework of public policies and measures developed during the pandemic, overcoming the traditional adult-centric views.

- The pandemic has affected and changed the lives and routines for young children, often with huge limitations on the children's vital need to play, move, and relate with their peers, and with reduced social contacts beyond the family.

- Children have the right to quality ECEC, which implies maintaining stable relationships with significant adults, caregivers, teachers and with their peers.

- The right to education during early childhood begins at birth and is linked to young children's right to maximum support to reach their full potential. For that, State parties and other actors must strengthen ECEC, assuring rich opportunities, even in this crisis.

- Young children learn by experiencing, playing, and exploring, along with other children and "significant others", using multiple languages and their body contact and movement to communicate with others.

- The educational policies must consider the importance of continuity of ECEC, through creative and wide proposals, strategies and materials; taking into account the need for play and movement of young children, they must not be exposed for long hours to screens, so that recommendations for the use of virtual tools to caregivers should be delivered.

- In response to the characteristics of the stage, ECEC develops methodologies focused on play, the involvement of the body as a way of experiencing, discovering, and investigating the environment, the manipulation of natural and cultural objects, and close human contact.

- The organization of the ECEC environments and scenarios is characterized by multifunctional activities provided with different materials, toys, furniture, allowing free movement, play, art expressions, recreation, and outdoor play, as well as hygienic practices, nurturing, and rest.

- Due to the characteristics of the early years and the ways in which they build their emotional and affective relationships with the educators and their peer 
group, gestures, hugs, physical contact, body language, and facial expression are needed.

- The principle of progressive autonomy implies that handwashing and other hygienic habits require time and patience for a respectful development.

\section{Based on the Above Considerations, State Parties Should}

- Include the consideration of children's perspectives in all matters that affect them. Their ideas, emotions, interests, and needs must be considered in the framework of public policies and measures developed during the pandemic and post-pandemic, overcoming traditional views focused on adults.

- Assume that the opening and operation of ECEC centers for children cannot depend solely on a vision focused on health or in response to economic interests. Decisions should consider the experience and knowledge of educators and families, as well as other related sectors.

- Build comprehensive solutions with intersectoral articulations to accompany and support families, protecting children's right to health, food security, recreation, and play, vital for their growth and development, preventing and intervening in situations of domestic violence and abuse.

- Provide a comprehensive protection of the rights, addressing special measures for the most vulnerable groups: children in poverty, inequality, famine and armed conflict; children on migration, displaced, living in camps or imprisoned, children affected by climate change, pollution, environmental degradation and natural disasters; children affected by racism, xenophobia and gender discrimination; children suffering from sexual or labor exploitation.

- Guide and train educators on distance education strategies and communication with families and children, both during the closure of the centers and in the implementation of protocols and new approaches in institutional projects for the reopening of institutions, ensuring the provision of the necessary materials and means.

- Guarantee young children their right to health, giving continuity to regular check-ups, vaccinations, and timely care, especially attending to recurrent eruptive and respiratory diseases during cold seasons, as well as implementing prevention and promotion strategies for health-based education.

- Consider the importance of a good use of available technologies, through creative and comprehensive proposals, strategies and materials, taking into account, that young children should not be exposed to screens for long hours and, on the other hand, that there are deep inequalities in the access of technology devices and internet connections.

\section{A NEW REALITY for Early Childhood}

Finally, the COVID-19 crisis has shown how connected we are worldwide. We cannot continue supporting this civilizing model, which has led us to an unprecedented human, social, educational, economic, and environmental global crisis. 
We must focus our responsibility on the present and future lives of our children, and this requires rebuilding the society we want. There is a possibility of creating a better world, but it needs collective and solidarity efforts based on the lessons of this crisis.

Children need a State, a family, and a civil society determined to promote an holistic and humanistic education and protect their dignity and rights.

OMEP wants to highlight the importance of creating a sustainable world for humanity and for the planet, and especially for children. We will continue working in solidarity to defend and maintain the progress made toward the achievement of the Sustainable Development Goals and SDG 4, convinced that education is the main tool for building a better world for all, leaving no one behind.

SIGNED By: OMEP EXECUTIVE COMMITTEE

******************************************************************** $* * *$

\section{Éducation et Garde de La Petite Enfance à L'époque de COVID-19}

Les premières années sont d'une importance vitale et les États parties doivent prendre des mesures spéciales pour protéger les enfants et promouvoir des conditions de vie adéquates basé sur l'intérêt supérieur de l'enfant, après la ratification de la Convention Internationale des Droits de l'Enfant (CIDE). En cette période dominée par la pandémie mondiale, il existe des risques supplémentaires pour les jeunes enfants. La pandémie n'est pas seulement un virus, c'est une énorme crise humaine et il est vérifié que les enfants vivant dans des conditions de pauvreté, d'extrême pauvreté et d'inégalité ont vu leur situation s'aggraver.

Il existe également des conséquences indirectes qui affectent profondément la vie et le développement des enfants, telles que les décès familiaux, la peur, l'accouchement, la faim, l'éloignement physique, la violence et les abus accrus, le manque de moments ludiques, le manque de mouvement, et une exposition excessive aux écrans.

Bien que le contexte et la vie quotidienne des jeunes enfants soient très différents, l'OMEP souhaite mettre en évidence certains points cruciaux au nom de l'intérêt supérieur de tous les enfants et de leur bien-être dans le monde.

Les États parties sont les gardiens des droits de l'enfant et ont le devoir d'appliquer les politiques nécessaires pour protéger et soutenir les enfants et les familles, en collaboration avec les autorités et organisations locales. La protection et d'éducation de la petite enfance (EPPE) jouent un rôle clé dans ce soutien.

- Les jeunes enfants sont des citoyens et les États parties devraient également tenir compte de leurs besoins spécifiques dans le contexte de l'éducation, des soins, de la santé, de l'économie et du soutien social.

- Les jeunes enfants nécessitent l'adoption de mesures spécifiques dans le cadre de la CIDE. Tous les enfants ont le droit de s'exprimer librement sur toutes les questions les concernant et ils doivent être entendus. Leurs idées, leurs émotions, leurs perspectives, leurs intérêts et leurs besoins doivent être pris en compte dans 
le cadre des politiques et mesures publiques élaborées pendant la pandémie, dépassant les points de vue traditionnels axés sur les adultes.

- La pandémie a affecté et changé la vie et les routines des jeunes enfants, imposant d'énormes limitations au besoin vital des enfants de jouer, de bouger et d'interagir avec avec leurs pairs, et réduisant les contacts sociaux à la famille.

- Les enfants ont droit à la qualité de la EPPE, ce qui implique de maintenir des relations stables avec les adultes significatifs, les soignants, les enseignants et leurs pairs.

- Le droit à l'éducation de la petite enfance commence à la naissance et est étroitement lié au droit des jeunes enfants à recevoir un soutien maximal afin de leur permettre d'atteindre leur plein potentiel. Pour ce faire, les États parties et les autres acteurs doivent faire face à l'ÉPÉE, et garantir de riches opportunités, même dans cette crise.

- Les jeunes enfants apprennent en expérimentant, en jouant et en explorant avec d'autres enfants et des "autres significatifs", en utilisant plusieurs langues et leur contact corporel et leurs mouvements pour communiquer avec les autres.

- Les politiques éducatives devraient tenir compte de l'importance de la continuité de l'ÉPÉE par le biais de propositions, de stratégies et de matériels créatifs et complets, en tenant compte de la nécessité de jouer et de bouger et ainsi ne pas exposer les jeunes enfants à des écrans pendant de longues heures, par conséquent, des recommandations pour l'utilisation d'outils virtuels devraient être diffusées auprès des soignants.

- En réponse aux caractéristiques de la stade, l'ÉPÉE développe des méthodologies centrées sur le jeu, la participation du corps comme moyen d'expérimenter, de découvrir et d'étudier l'environnement, la manipulation d'objets naturels et culturels, et le contact humain étroit.

- L'organisation des environnements et des scénarios de l'ÉPÉE se caractérise par des activités multifonctionnelles dotées de différents matériaux, jouets, meubles, qui permettent la libre circulation, le jeu, les expressions artistiques, les loisirs et les jeux en plein air, ainsi que les pratiques hygiéniques, nourrissement et repos.

- En raison des caractéristiques des premières années et la façon dont ils construisent leurs relations émotionnelles et affectives avec les éducateurs et leur groupe de pairs, des gestes, des câlins, des contacts physiques, le langage corporel et l'expression faciale sont nécessaires.

- Le principe de l'autonomie progressive implique que le lavage des mains et d'autres habitudes hygiéniques nécessitent du temps et de la patience pour un développement respectueux.

\section{Sur la base de les considérations précédentes, les États parties devraient}

- Inclure la prise en compte du point de vue des enfants dans toutes les questions qui les concernent. Leurs idées, émotions, intérêts et besoins doivent être pris en compte dans le cadre des politiques et mesures publiques développées pendant la pandémie et la post-pandémie, dépassant les visions traditionnelles centrées sur les adultes. 
- Assumer que l'ouverture et le fonctionnement des centres de l'ÉPÉE pour filles et garçons ne peuvent dépendre uniquement d'une vision centrée sur les aspects sanitaires ou en réponse à des intérêts économiques, les décisions doivent tenir compte de l'expérience et des connaissances des éducateurs et des familles, ainsi que d'autres secteurs connexes.

- Élaborer des solutions intégrales avec des articulations intersectorielles pour accompagner et soutenir les familles, protéger le droit des enfants à la santé, à la sécurité alimentaire, aux loisirs et au jeu, qui sont vitales pour leur croissance et leur développement, afin de prévenir et d'intervenir dans les situations de violence domestique et d'abus.

- Fournir une protection complète des droits, en prévoyant des mesures spéciales pour les groupes les plus vulnérables: confrontés à la pauvreté, aux inégalités, à la famine et aux conflits armés; les enfants en migration, déplacés, vivant dans des camps ou emprisonnés; les enfants affectés par le changement climatique, la pollution, la dégradation de l'environnement et les catastrophes naturelles; les enfants touchés par le racisme, la xénophobie et la discrimination; enfants souffrant d'exploitation sexuelle ou aux travaux forcés.

- Guider et former les éducateurs sur les stratégies d'enseignement à distance et sur la communication avec les familles et les enfants, à la fois lors de la fermeture des centres et dans la mise en œuvre de protocoles et de nouvelles approches dans les projets institutionnels pour la réouverture des institutions, en assurant la fourniture du matériel et des moyens nécessaires.

- Garantir aux jeunes enfants leur droit à la santé, en assurant la continuité des contrôles réguliers, des vaccinations et des soins en temps opportun, en particulier en cas de maladies éruptives et respiratoires récurrentes pendant les saisons froides, ainsi qu'en mettant en œuvre des stratégies de prévention et de promotion de la santé basée sur l'éducation.

- Considérer l'importance d'une bonne utilisation des technologies disponibles, à travers des propositions, stratégies et matériels créatifs et complets, en tenant compte, d'une part, que les jeunes enfants ne doivent pas être exposés à des écrans pendant de longues heures et, d'autre part, qu'il existe des inégalités d'accès aux appareils technologiques et aux connexions Internet.

\section{Une NOUVELLE RÉALITÉ pour la petite enfance}

Enfin, la crise du COVID-19 a montré à quel point nous sommes connectés dans le monde. Il est clair que nous ne pouvons pas continuer à soutenir ce modèle civilisateur, qui nous a conduits à une crise mondiale sans précédent sur les plans humain, social, éducatif, économique et environnemental. Nous devons concentrer notre responsabilité sur la vie actuelle et future de nos enfants, ce qui nécessite une réflexion urgente sur la société que nous voulons pour nos enfants.

Il est possible de créer un monde meilleur, mais cela nécessitera un effort collectif et solidaire qui tirera parti des leçons de cette crise. 
Les enfants ont besoin d'un État, d'une famille, et d'une société civile déterminée à promouvoir d'une éducation holistique et humaniste et protéger leur dignité et leurs droits.

L'OMEP veut souligner l'importance de créer un monde durable, pour l'humanité et pour la planète et spécialement pour les enfances. Nous continuerons à travailler en solidarité pour défendre et maintenir les progrès accomplis vers la réalisation des Objectifs de Développement Durable et de l'ODD 4, convaincu que l'éducation est le principal outil pour construire un monde meilleur pour tous, pour ne laisser personne de côté.

SIGNÉ PAR: COMITÉ EXÉCUTIF DE L'OMEP

******************************************

\section{Educación y Cuidado de la Primera Infancia en Tiempos de COVID-19}

Los primeros años son de vital importancia y los Estados Parte deben tomar medidas especiales para proteger a los niños y niñas, y promover buenas condiciones de vida basadas en el interés superior del niño, de acuerdo con lo dispuesto por la Convención sobre los Derechos del niño (CDN). En este tiempo dominado por la pandemia mundial hay riesgos adicionales para los niños y niñas pequeños. La pandemia no es sólo un virus, es una enorme crisis humanitaria y se verifica que los niños y niñas que viven en condiciones de pobreza, pobreza extrema y desigualdad han empeorado su situación.

También hay consecuencias indirectas que afectan profundamente la vida y el desarrollo de los niños y niñas como las muertes familiares, el miedo, el confinamiento, el hambre, el distanciamiento físico, el aumento de la violencia y el abuso, la falta de momentos para el juego y el movimiento, y la exposición excesiva a las pantallas.

Aunque el contexto y la vida cotidiana de los niños y niñas pequeños son muy diferentes, OMEP quiere destacar ciertos puntos cruciales en nombre del interés superior de todos los niños y niñas, y su bienestar en todo el mundo.

Los Estados Parte son garantes del cumplimiento de los Derechos del Niño y tienen el deber de aplicar las políticas necesarias para proteger y apoyar a los niños, las niñas y las familias, en colaboración con las autoridades y organizaciones locales. La Atención y Educación de la Primera Infancia (AEPI) desempeña un papel fundamental en este apoyo.

- Las niñas y niños pequeños son ciudadanos y los Estados Parte deben considerar también sus necesidades específicas en el contexto de la educación, el cuidado, la salud, la economía y el apoyo social.

- Las niñas y niños pequeños requieren la adopción de medidas específicas en el marco de la CDN. Cada niño y niña tiene derecho a expresar sus puntos de vista libremente en todos los asuntos que les afecten, y a ser tenidos en cuenta. Sus ideas, emociones, perspectivas, intereses y necesidades deben ser consideradas en el marco de las políticas y medidas públicas desarrolladas durante la pandemia, superando los puntos de vista tradicionales centrados en los adultos. 
- La pandemia ha afectado y cambiado las vidas y rutinas de las niñas y niños pequeños, a menudo con enormes limitaciones en la necesidad vital de jugar, moverse y relacionarse con sus pares, y con contactos sociales reducidos más allá de la familia.

- Los niños y niñas tienen derecho a una AEPI de calidad, lo que implica mantener relaciones estables con adultos significativos, cuidadores, maestros y con sus pares.

- El derecho a la educación durante la primera infancia comienza al nacer y está estrechamente relacionado con el derecho de las niñas y niños pequeños a recibir el máximo apoyo para alcanzar todo su potencial. Para ello, los Estados Parte y otros actores tienen que fortalecer la AEPI garantizando oportunidades ricas, incluso en esta crisis.

- Las niñas y niños pequeños aprenden experimentando, jugando y explorando, junto con otros niños y niñas y "otros significativos", utilizando múltiples lenguajes y su contacto corporal y movimiento para comunicarse con los demás.

- Las políticas educativas deben considerar la importancia de la continuidad de la AEPI, a través de propuestas, estrategias y materiales creativos y amplios; teniendo en cuenta la necesidad del juego y del movimiento de las niñas y niños pequeños, y que ellos no deben estar expuestos durante largas horas a las pantallas, por eso se deben difundir entre los cuidadores recomendaciones para el uso de herramientas virtuales.

- En respuesta a las características de la etapa, la AEPI desarrolla metodologías centradas en el juego, la participación del cuerpo como una forma de experimentar, descubrir e investigar el medio ambiente, la manipulación de objetos naturales y culturales, y el contacto humano cercano.

- La organización de los entornos y escenarios de la AEPI se caracteriza por actividades multifuncionales provistas de diferentes materiales, juguetes, muebles que permiten la libre circulación, el juego, las expresiones artísticas, la recreación y el juego al aire libre, así como las prácticas higiénicas, la crianza y el descanso.

- Debido a las características de los primeros años y los modos en que construyen sus relaciones emocionales y afectivas con sus educadores y con su grupo de pares, son necesarios los gestos, los abrazos, el contacto físico, el lenguaje corporal y la expresión facial. El principio de autonomía progresiva implica que el lavado de manos y otros hábitos higiénicos requieren tiempo y paciencia para un desarrollo respetuoso.

\section{Sobre la base de las consideraciones anteriores, los Estados Parte deberían}

- Incluir la consideración de las perspectivas de los niños y niñas en todos los asuntos que les afecten. Sus ideas, emociones, intereses y necesidades deben ser tomadas en cuenta en el marco de las políticas públicas y las medidas desarrolladas durante la pandemia y la post pandemia, superando los puntos de vista tradicionales centrados en los adultos.

- Asumir que la apertura y funcionamiento de los centros de AEPI para niñas y niños no puede depender únicamente de una visión centrada en aspectos sani- 
tarios o en respuesta a intereses económicos. Las decisiones deben considerar la experiencia y saberes de los educadores y las familias, así como otros sectores relacionados.

- Construir soluciones integrales con articulaciones intersectoriales para acompañar y apoyar a las familias, protegiendo el derecho de los niños y la niñas a la salud, la seguridad alimentaria, la recreación y el juego, vitales para su crecimiento y desarrollo, previniendo e interviniendo en situaciones de violencia doméstica y abuso.

- Proporcionar una protección integral de los derechos, abordando medidas especiales para los grupos más vulnerables: niños y niñas en situación de pobreza, desigualdad, hambrunas y conflictos armados; niños y niñas en migración, desplazados, que viven en campamentos o encarcelados; niños y niñas afectados por el cambio climático, la contaminación, la degradación del medio ambiente y los desastres naturales; niños y niñas afectados por el racismo, la xenofobia y la discriminación de género; niños y niñas que sufren de explotación sexual o laboral.

- Orientar y capacitar a los educadores sobre estrategias de educación a distancia y comunicación con las familias y los niños y niñas, tanto durante el cierre de los centros, como en la implementación de los protocolos y nuevos enfoques en los proyectos institucionales para la reapertura de las instituciones, asegurando la provisión de los materiales y medios necesarios.

- Garantizar a las niñas y niños pequeños su derecho a la salud, dando continuidad a los controles periódicos, las vacunas y la atención oportuna, especialmente atendiendo enfermedades eruptivas y respiratorias recurrentes durante las estaciones de frío, como así también, implementando estrategias de prevención y promoción de la salud basadas en la educación.

- Considerar la importancia de un buen uso de las tecnologías disponibles, a través de propuestas, estrategias y materiales creativos y amplios, teniendo en cuenta, por un lado que los niños y niñas pequeños no deben exponerse durante largas horas a las pantallas, y por otro que existen profundas desigualdades en el acceso de dispositivos tecnológicos y conexiones a internet.

\section{Una NUEVA REALIDAD para la primera infancia}

Por último, la crisis del COVID-19 ha demostrado lo conectados que estamos en todo el mundo. Está claro que no podemos continuar sosteniendo este modelo civilizatorio que nos ha llevado a una inédita crisis humana, social, educativa, económica y ambiental global. Debemos centrar la mirada en nuestra responsabilidad sobre las vidas presentes y futuras de nuestros niños y niñas, y para ello se requiere un urgente replanteamiento sobre la sociedad que queremos.

Existe la posibilidad de crear un mundo mejor, pero requerirá un esfuerzo colectivo y solidario que capitalice las lecciones de esta crisis.

Los niños y niñas necesitan de un Estado, de una familia, de una sociedad civil decidida a promover una educación holística y humanista, y la protección de su dignidad y sus derechos. 
La OMEP quiere subrayar la importancia de crear un mundo sostenible, para la humanidad, para el planeta, y especialmente para las infancias. Continuaremos trabajando en solidaridad para defender y mantener el progreso hecho en el logro de los Objetivos de Desarrollo Sostenible y el ODS 4, convencidos y convencidas de que la educación es la principal herramienta para construir un mundo mejor para todos y todas, sin dejar a nadie atrás.

FIRMADO POR: EL COMITÉ EJECUTIVO MUNDIAL DE OMEP

Publisher's Note Springer Nature remains neutral with regard to jurisdictional claims in published maps and institutional affiliations. 